\title{
Analyst
}

ARTICLE

\section{Dielectric Spectroscopy Platform to Measure MCF10A Epithelial Cell Aggregation as a Model for Spheroidal Cell Cluster Analysis}

Received 00th January 20xx Accepted 00th January 20xx DOI: $10.1039 / x 0 x \times 00000 x$

www.rsc.org/

\author{
K. L. Heileman ${ }^{\mathrm{a}}$ and M. Tabrizian ${ }^{\mathrm{a}, \mathrm{b}}$
}

3-dimensional cell cultures are more representative of the native environment than traditional cell cultures on flat substrates. As a result, 3-dimensional cell cultures have emerged as a very valuable model environment to study tumorigenesis, organogenesis and tissue regeneration. Many of these models encompass the formation of cell aggregates, which mimic the architecture of tumor and organ tissue. Dielectric impedance spectroscopy is a non-invasive, label free and real time technique, overcoming the drawbacks of established techniques to monitor cell aggregates. Here we introduce a platform to monitor cell aggregation in a 3-dimensional extracellular matrix using dielectric spectroscopy. The MCF10A breast epithelial cell line serves as a model for cell aggregation. The platform maintains sterile conditions during the multiday assay while allowing continuous dielectric spectroscopy measurements. The platform geometry optimizes dielectric measurements by concentrating cells within the electrode sensing region. The cells show a characteristic dielectric response to aggregation which corroborates with finite element analysis computer simulations. By fitting the experimental dielectric spectra to the Cole-Cole equation, we demonstrated that the dispersion intensity $\Delta \varepsilon$ and the characteristic frequency $f_{c}$ are related to cell aggregate growth. In addition, microscopy can be performed directly on the platform providing information about cell position, density and morphology. This platform could yield many applications for studying the electrophysiological activity of cell aggregates.

\section{Introduction}

In vitro monitoring of cell aggregation has important applications for studying tumorigenesis, organogenesis and tissue regeneration. Since cell aggregates are 3-dimensional cultures, they better emulate the physiological cell environment, intercellular interactions and cell-matrix interactions than 2-dimensional cell cultures on flat substrates 1. The advantages of 3-dimensional cultures are illustrated in in vitro models of tumours such as breast cancer models. For example, the differences in morphology, cell polarization, matrix interactions and growth rate between normal breast tissues and tissues with various levels of malignancy become significantly more discernible in 3-dimensional assays 2, 3 . Therefore, 3-dimensional assays provide a better representative for analysis of tumour initiation, metastasis and drug response. Cell aggregates have proven to be relevant 3dimensional cell culture models. These models include spheroids composed of malignant cells to mimic tumours and embryonic bodies that are used to study morphogenesis and tissue formation ${ }^{4,5}$. Spheroids have also been developed from various tissues including liver, cartilage and cardiac tissue ${ }^{6}$. Another application for cell aggregates lies in developing artificial tissues for transplantation and regenerative medicine.

\footnotetext{
a. Biomedical Engineering Department, McGill University, Montreal, Quebec H3A

2B4, Canada. Tel.: +1-514-398-8129. Fax: +1-514-398-7461

b. Faculty of Dentistry, McGill University, Montreal, Quebec H3A 2B4, Canada.
}

A notable example is the aggregation of cells into pseudo-islets, which mimic many of the features of pancreatic islets of Langerhans, including insulin expression and secretion 7-9. Pseudo-islets may potentially help to overcome shortages of cadaveric donor islets for transplantation in type 1 diabetic patients.

Detailed analysis of intact cell aggregate tissue typically necessitates confocal, multiphoton and light sheet fluorescent microscopy ${ }^{10-12}$. However, the drawbacks of these techniques are that they are highly invasive and require the use of costly fluorescent reporter probes as well as complex sample preparation. To yield information about phenotype and differentiation, gene expression analysis of cell aggregates can be performed with RT-PCR amplification followed by gene microarrays ${ }^{11}$. Nevertheless, gene expression analysis typically entails RNA extraction from the cells, making continuous monitoring impossible. Therefore, rapid, real time and noninvasive monitoring techniques would benefit analysis of 3dimensional assays in terms of ease of use, costs and analysis time ${ }^{13}$.

Dielectric impedance spectroscopy is a rapid, non-invasive and real time technique for cell monitoring. This technique is particularly beneficial for monitoring cell aggregation - and respective cellular processes - which could last up to several days. Hence, dielectric spectroscopy provides a viable method to perform continuous real-time and non-invasive measurements without perturbing cell culture conditions. 
The MCF10A epithelial breast cell line was used in the proof-ofconcept methodology for sensing cell aggregation using dielectric spectroscopy. This cell line has served as an in vitro model of breast tissue morphogenesis and cancer tumorigenesis 14, 15. The in vitro tumorigenesis model of epithelial breast cells encompasses aggregation of dispersed cells into clusters that lack a central lumen ${ }^{14}$. A platform was fabricated to carry out dielectric spectroscopy measurements, which includes an electrode bottomed well containing MCF10A cells suspended in a matrigel extracellular matrix. Dielectric spectroscopy measurements of aggregates were taken periodically to investigate the effects of cell aggregation on their dielectric response. At the end of the assay, the morphology of the tissue was confirmed with confocal microscopy imaging.

\section{Materials and Methods}

\section{Numerical Calculation of Cell Aggregate Dielectric Response by Finite Element Analysis (FEA)}

The dielectric response to cell aggregation on the platform was simulated with numerical calculations using the AC/DC module on the finite element analysis software COMSOL Multiphysics (Sweden). The simulations provided validation for the chosen platform design, which comprised a well holding an extracellular matrix/cell suspension and bottomed with interdigitated electrodes (figure 1). The simulation geometry included a sphere in an extracellular matrix and two planar electrodes (figure 2A). These electrodes represent only a single electrode pair from the overall interdigitated electrode setup. The sphere represents a cellular aggregate, which for simplicity was given a spherical geometry with a single conducting core surrounded by a non-conducting membrane. On the platform, the electrodes must be able to detect large cell aggregates that are relatively distant from the electrode surface. Therefore, in order to represent experimental conditions, the sphere in the simulation was suspended above the electrodes by a $200 \mu \mathrm{m}$ gap. We measured the dielectric properties of the matrigel extracellular matrix beforehand and found them to be similar to assay media (the composition of this media is covered in the cell aggregation assay section). Hence in the simulation, the extracellular matrix was given typical dielectric properties for cell media. The cytoplasm and extracellular media were assigned a permittivity and conductivity of $\varepsilon_{c}=80$ and $\kappa_{c}=1$ respectively, and the cell membrane was assigned a permittivity and conductivity of $\varepsilon_{m}=5$ and $\kappa_{m}=0$ respectively. These electrical parameters are typical for biological cells ${ }^{16}$. To generate an

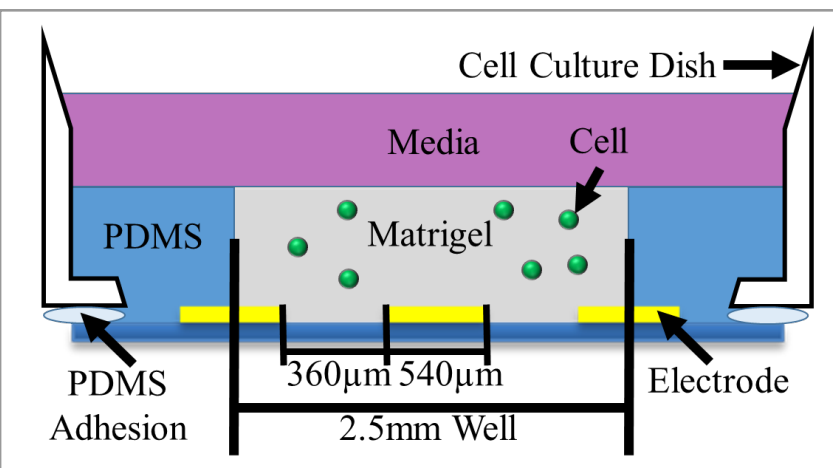

Figure 1 Dielectric spectroscopy platform for measuring cell aggregation. Sideview schematic of the assembled platform showing the position of the MCF10A cells and matrigel during the assay.

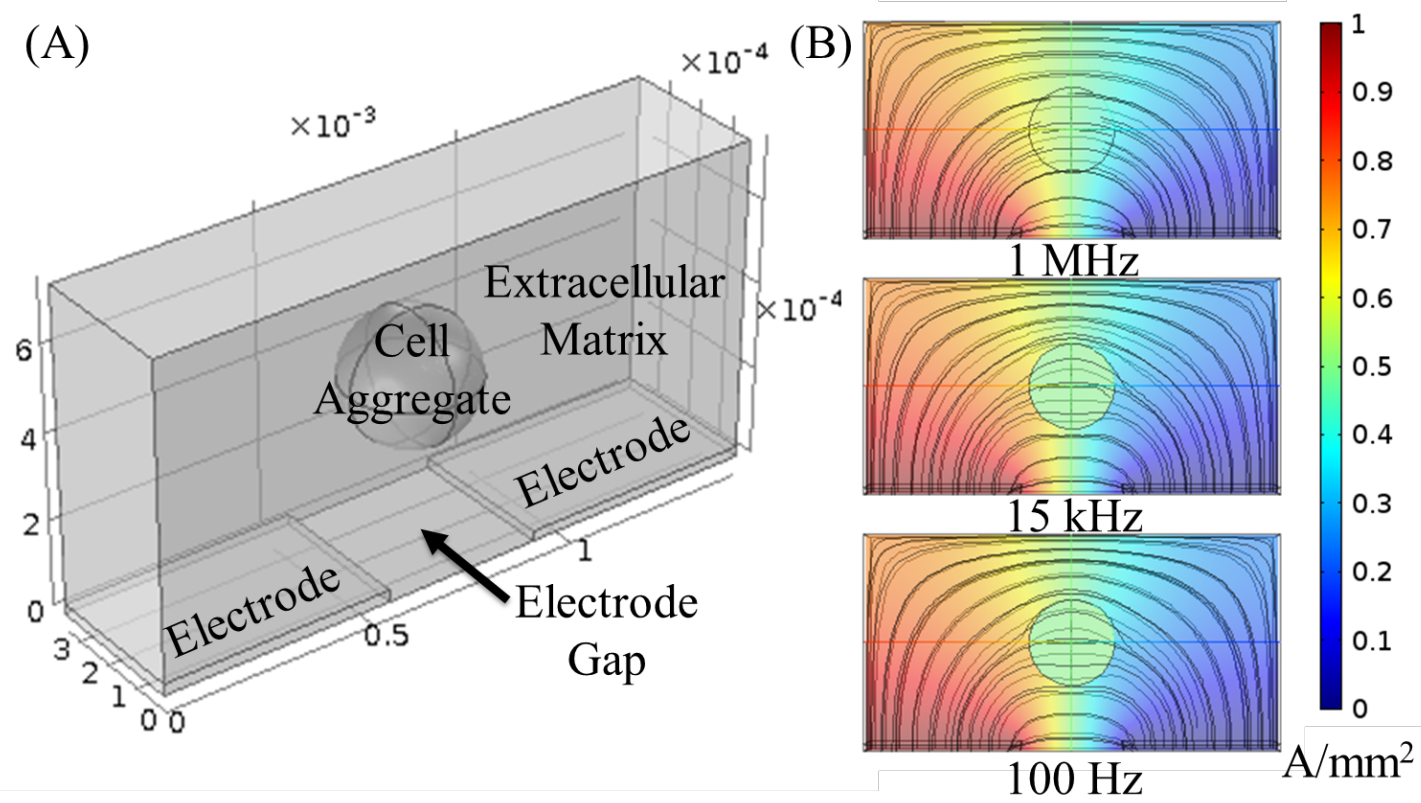

Figure 2 FEA simulation of the dielectric response to cell aggregation (A) The simulation geometry is composed of a cell aggregate, the extracellular media and two planar electrodes. (B) Current density for the $300 \mu \mathrm{m}$ diameter cell showing, for higher frequencies, the penetration of the electric field across the cell membrane and into the cytoplasm. 
electric field, a potential difference of $1 \mathrm{~V}$ was applied to both electrodes. The frequency of the electric field was swept from $100 \mathrm{~Hz}$ to $10 \mathrm{MHz}$. The diameter of the cell was varied between 100,200 and $300 \mu \mathrm{m}$ to simulate the growth of the cell clusters during aggregation.

\section{Electrode Design and Microfabrication}

Electrode chrome masks were designed with an interdigitated electrode layout (10 digits, $540 \mu \mathrm{m}$ digit width and $360 \mu \mathrm{m}$ inter-digit space). This electrode configuration ensures $80 \%$ of the signal passes within $450 \mu \mathrm{m}$ above the substrate ${ }^{17}$, so that cells suspended above the electrodes are detected. Electrode microfabrication was achieved using photolithography in a cleanroom environment. LOR5B ${ }^{\mathrm{TM}}$ (Microchem, Newton, MA) was spin-coated at $1000 \mathrm{rpm}$ for 30 seconds on a glass wafer substrate and then baked at $180^{\circ} \mathrm{C}$ for 5 minutes. On top the LOR5B $^{\text {TM }}$ layer, Microposit ${ }^{\mathrm{TM}}$ S1813 ${ }^{\mathrm{TM}}$ (Shipley, Marlborough, MA) was spin-coated at $3950 \mathrm{rpm}$ for 30 seconds and baked at $115^{\circ} \mathrm{C}$ for 1 minute. A chrome mask was used to pattern the photoresist under ultraviolet (UV) exposure $\left(124.3 \mathrm{~mJ} / \mathrm{cm}^{2}\right)$. The substrate was then developed in microposit MFVR-319 (Shipley) for 50 seconds, washed in deionized water and air dried. A titanium adhesion layer $(10 \mathrm{~nm})$ and a gold layer $(50$ $\mathrm{nm}$ ) were deposited using electron beam evaporation. The photoresist was lifted off using Microposit Remover 1165 (Shipley) at $70^{\circ} \mathrm{C}$ to yield the final electrode layout. Finally, the glass wafers were removed from the cleanroom and then cut into single electrode pieces using a diamond saw.

\section{Dielectric Spectroscopy Platform Assembly}

To create a sterile environment for continuous dielectric measurement of cells, a cell culture chamber was built on top of the electrode. The assembled platform is shown in figure 1. The glass bottoms from glass bottomed culture dishes (MatTek) were manually removed, leaving a circular void with a $14 \mathrm{~mm}$ diameter. Polydimethylsiloxane (PDMS) was prepared by mixing the base and curing agent at a 10:1 weight ratio. PDMS was then spread on the bottom of the culture dishes to facilitate adhesion to the electrode substrate, with the interdigitated electrodes positioned inside the culture dish void. The PDMS was then cured by placing the assembly in an oven at $70^{\circ} \mathrm{C}$ for 1 hour. A second PDMS layer was created by pouring a 10:1 weight mixture of PDMS and curing agent on top of the electrodes. A standing $5 \mathrm{ml}$ pipette was positioned in the PDMS layer to define the well. The pipette was carefully aligned to ensure that the well bottom contains at least 2 electrode pairs. The PDMS was cured in an oven at $70^{\circ} \mathrm{C}$ for 1 hour, after which the pipette was removed, yielding a well with a diameter of $2.5 \mathrm{~mm}$. This PDMS layer masks most of the electrode surface, reducing the contribution of the high conductivity media to the dielectric spectra. The well also concentrates cells in a small region, improving the platform sensitivity. Without the PDMS layer, the cell dielectric response is un-resolvable as determined from previous experiments, because the dielectric properties of the extracellular media dominate. Moreover, the well decreases the amount of matrigel required for the assay, reducing costs.

\section{MCF10A Cell Culture}

MCF10A cells were acquired from American Type Culture Collection (ATCC). A growth medium was prepared from $500 \mathrm{ml}$ DMEM F/12 media containing $25 \mathrm{ml}$ horse serum (ATCC), $100 \mu$ epidermal growth factor (EGF), $250 \mu$ hydrocortisone, $50 \mu \mathrm{l}$ cholera toxin, $500 \mu \mathrm{l}$ insulin and $5 \mathrm{ml}$ penicillin streptomycin (pen/strep). Cells were grown as a monolayer in a cell culture flask containing growth medium. For passaging, 0.05\% trypsinEDTA was used to re-suspend cells. The cells were then washed with medium containing $400 \mathrm{ml}$ DMEM/F12, $100 \mathrm{ml}$ horse serum (ATCC) and $5 \mathrm{ml}$ pen/strep. The cells were never allowed to reach confluency.

\section{Cell Aggregation Assay}

The assay (layout shown in figure 1) was adapted from a 3 dimensional culture of MCF10A cells in matrigel ${ }^{15}$. Assay media was prepared with the same ingredients as growth media, except with $10 \mathrm{ml}$ horse serum (ATCC) and $25 \mu \mathrm{l} \mathrm{EGF}$. The day before cell seeding, MCF10A cells were passaged while the matrigel (BD biosciences) was left to thaw overnight at $4^{\circ} \mathrm{C}$ on ice. On the day of cell seeding, the devices were cleaned with soap and sterilized using $70 \%$ ethanol followed by a 1 hour UV light treatment in a cell culture hood. Using pre-chilled pipettes, MCF10A cells were re-suspended in cold matrigel. Around $15 \mu \mathrm{l}$ of the cell/matrigel suspension was deposited into the device well. The devices were then placed in an incubator $\left(37^{\circ} \mathrm{C}\right.$ and $5 \%$ $\mathrm{CO}_{2}$ ) for 20 minutes to allow the matrigel to solidify. Assay media was then added to the devices and changed in this fashion every 2 days. The cells were kept in the same incubator and the assay lasted for a total of 7 days. One device was kept cell-free to serve as a control. Like the other devices, the control device had a well containing matrigel, was kept in the incubator and had the same media change schedule. The assays were inspected daily with a microscope.

\section{Immunofluorescent (IF) Solution Preparation}

10xPBS:glycine was prepared by adding $\mathrm{NaCl}(7.6 \mathrm{~g}), \mathrm{Na}_{2} \mathrm{HPO}_{4}$ $(1.88 \mathrm{~g}), \mathrm{NaH}_{2} \mathrm{PO}_{4}(0.414 \mathrm{~g})$ and glycine $(7.5 \mathrm{~g})$ to water $(100 \mathrm{ml})$. 10xIF-wash was prepared by adding $\mathrm{NaCl}(7.6 \mathrm{~g}), \mathrm{Na}_{2} \mathrm{PO}_{4}(1.88$ g), $\mathrm{NaH}_{2} \mathrm{PO}_{4}(0.414 \mathrm{~g}), \mathrm{NaN}_{3}(0.5 \mathrm{~g})$, bovine serum albumin (Jackson ImmunoResearch Laboratories) (1 g), Triton X-100 (2 $\mathrm{ml}$ ) and Tween-20 $(0.5 \mathrm{ml})$ to water $(100 \mathrm{ml})$. The $\mathrm{pH}$ of the 10xPBS:glycine wash and the 10xIF wash was adjusted to 7.4 followed by filter sterilization.

\section{MCF10A Cell Immunostaining}

MCF10A cells for immunofluorescent imaging were cultured in matrigel spread on top an electrode-free glass bottomed MatTek dish. The cells were acquired from the same flask as the cells subject to dielectric measurements and were seeded in matrigel on the same day. These cells were kept in an incubator $\left(37^{\circ} \mathrm{C}\right.$ and $\left.5 \% \mathrm{CO}_{2}\right)$ and were replenished with fresh assay media every 2 days. Therefore, the experimental conditions were the same for cells subject to dielectric measurements and cells for 
immunofluorescent imaging. On day 7 of the assay, these cells were fixed in 5\% formalin for 30 minutes and then stored in PBS at $4^{\circ} \mathrm{C}$. In preparation for immunofluorescent staining, the cells were first rinsed 3 times, 10 minutes each, in 1xPBS:glycine and then permeabilized for 10 minutes with $0.5 \%$ Triton $X-100$ in PBS. The cells were then blocked in goat serum (Invitrogen) for 1.5 hours. The staining was continued in the dark to protect fluorescent labels from light. The cells were incubated with Alexa Fluor 594 phalloidin (Invitrogen) for 1 hour. Afterwards, cells were rinsed 3 times, 20 minutes each, with 1xIF wash. Subsequently, cells were stained with Hoechst 33342 (1:1000) in PBS for 10 minutes. Finally, the cells were mounted with a mounting solution, capped with a coverslip and stored at $4^{\circ} \mathrm{C}$ until imaging with the Zeiss LSM 510 confocal system.

\section{Dielectric Spectroscopy Measurements}

Dielectric measurements were taken with the Agilent 4294A impedance analyser (Agilent, Palo Alto, CA). The spectra were recorded using 201 points/spectra at a frequency range of 40 $\mathrm{Hz}-10 \mathrm{MHz}$ and at amplitude of $100 \mathrm{mV}$. The device was interfaced with spring loaded pins that via shielded cables connected one electrode to the impedance analyser's high voltage/high current terminal, while the other electrode was connected to the low voltage/low current terminal. The sample was kept in an incubator $\left(37^{\circ} \mathrm{C}\right.$ and $\left.5 \% \quad \mathrm{CO}_{2}\right)$ during measurements. Each dielectric measurement of cells was subtracted from the dielectric spectra of the identical device without cells. This operation yielded differential spectra which reduces the effects of the electrode double layer capacitance and reveals the dielectric contribution of the cells. The spectra were then fit to the Cole-Cole model of dielectric relaxation ${ }^{18}$,

$$
\varepsilon^{*}=\varepsilon_{h}+\frac{\Delta \varepsilon}{1+\left(\frac{f}{f_{c}}\right)^{\beta}}+\frac{\kappa_{l}}{i \varepsilon_{0} 2 \pi f}
$$

where $\varepsilon^{*}$ is the complex permittivity, $\Delta \varepsilon$ is the intensity of the dispersion, $\varepsilon_{0}$ is the permittivity of a vacuum, $f_{c}$ is the characteristic frequency, $B$ is the Cole-Cole parameter $(0<B<1)$, $\mathrm{i}^{2}=-1, \kappa_{l}$ is the low frequency limit of conductivity and $f$ is the frequency of the applied field. The dielectric spectra were fitted to the Cole-Cole equation using the Levenberg-Marquardt least squares algorithm in MATLAB.

\section{Statistical Analysis}

The number of replicates for the cell aggregation assay was $n=3$. For each time point analysed in the dielectric spectra, the relative standard deviation was below $5 \%$. For graphical aesthetics, only averaged data points are presented. Data fitting to the Cole-Cole equation to yield dielectric parameters had an $\mathrm{R}^{2}>0.99$. One-tailed paired student's t-tests were used to identify statistically significant differences in dielectric parameters between subsequent days. A Bonferroni correction was applied, and as such, differences were considered significant for $\mathrm{p} \leq 0.01$.

\section{Results}

\section{FEA Simulation of Dielectric Response to Aggregating Cells}

The simulation yielded capacitance spectra plots in response to varying the cell aggregate diameter sizes between 100, 200 and $300 \mu \mathrm{m}$ (figure 3 ). The capacitance spectra contained a single $\beta$ dispersion which was caused by polarization across the nonconducting cell membrane. Increasing the cell aggregate diameter raised the capacitance spectra below $1 \mathrm{GHz}$ and therefore increased the dispersion intensity $\Delta \varepsilon$, a useful dielectric parameter related to aggregate size. The simulation also showed that at frequencies around $1 \mathrm{MHz}$ and higher, the electric field penetrates into the cytoplasm of the cell (figure 2B). This represents the shorting of the low conductivity

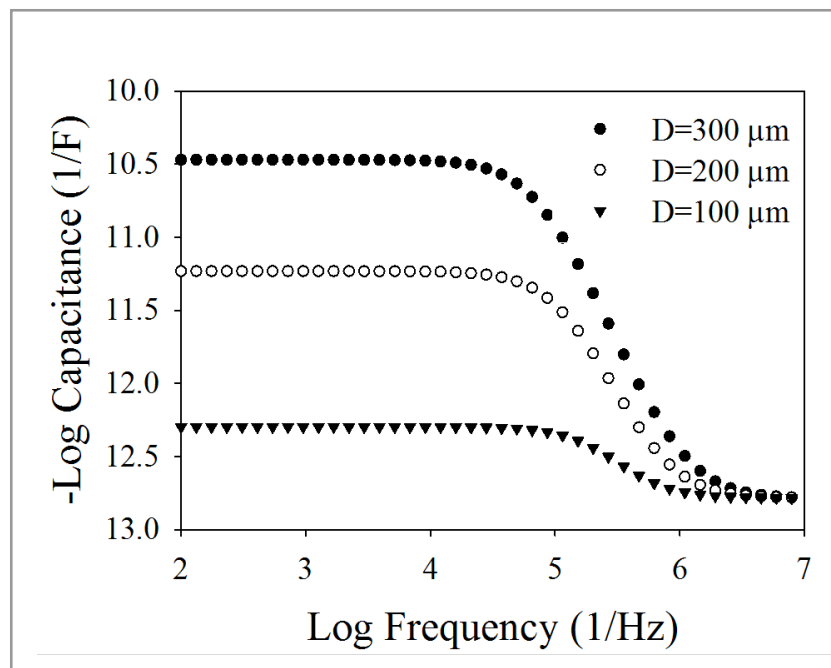

Figure 3 Capacitance spectra generated from the FEA simulation of cell aggregation. Capacitance spectra variation corresponding to the cell diameters $D$ of 100,200 and $300 \mu \mathrm{m}$.

membrane that occurs at higher frequencies, yielding information about the dielectric properties of the cell cytoplasm. The $\beta$ dispersions in the simulation only occurred when the volume fraction of the cell aggregate compared to the surrounding media was sufficiently high. Keeping this in mind, in the experimental setup, the cells had to be seeded at a sufficient volume fraction in order to resolve the cell dielectric response. Therefore, the platform includes a well to concentrate the cells into a smaller region.

\section{Cell Aggregation During the Assay}

Over the 7-day assay period, the single MCF10A cells formed aggregates. These aggregates lacked any discernible organization or central lumen when observed with light microscopy. The morphology and behaviour of these aggregates contrasts with typical MCF10A cell organogenesis in matrigel, which is characterized by the formation of growth arrested cystic structures ${ }^{15}$. Cystic structures are composed of cells exhibiting apical-basal polarity, with opposite ends of the cells in contact with the lumen of the cyst and the basement membrane, respectively. This divergence from normal organogenesis suggests that the aggregates were exhibiting a more tumorigenic phenotype ${ }^{14}$. Because of their particular 

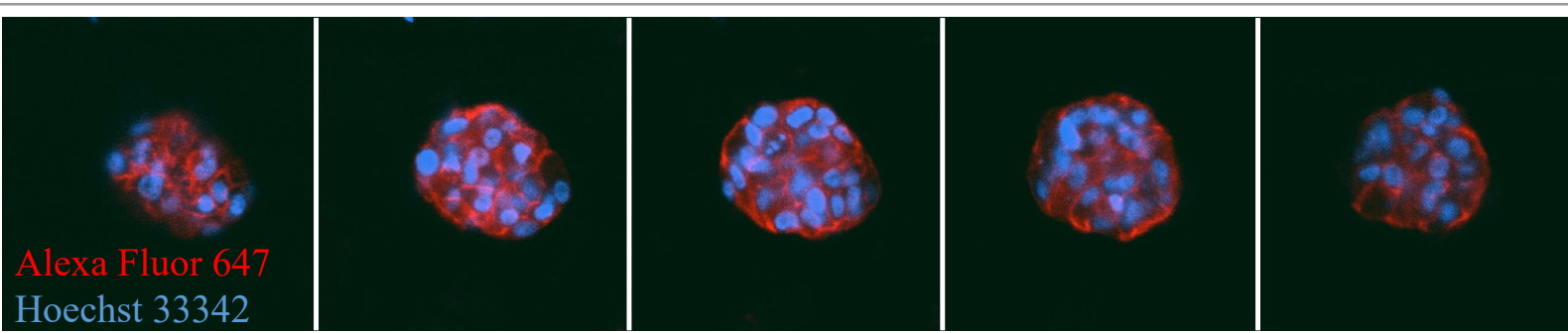

Figure 4 Confocal images of MCF10A aggregates on day 7 of the assay. These images represent different slices of the aggregate in the z-direction, with a $7.5 \mu \mathrm{m}$ gap between slices. The dimensions of each slice are $135.5 \mu \mathrm{m} \times 135.5 \mu \mathrm{m}$. The cytoskeleton is shown in red with Alexa Fluor 594, while the nuclei are stained blue with Hoechst 33342

behaviour, the MCF10A cells in this assay served as a model of cell aggregation.

\section{Immunofluorescent Confocal Images of Cell Aggregates}

The observations made with light microscopy regarding the 3dimensional morphology of the MCF10A cell aggregates were confirmed with confocal microscopy. A confocal image is displayed for a cell aggregate that was fixed on day 7 of the assay in figure 4. Confocal imaging showed that the cell aggregates lack a central lumen. The tissue was thick enough to allow acquisition of numerous optical slices of different cell layers. Within the aggregates, the cells were packed together without any apparent organization, demonstrating the loss of ability of the cells to maintain polarity.

\section{Dielectric Properties in Response to Cell Aggregation}

For the selected days of the assay, after subtracting the dielectric spectra of a cell sample from that of a cell-free well, a spectrum with a single $\beta$ dispersion was obtained (figure $5 A$ ). This $\beta$ dispersion is typical for cells in suspension and is analogous to that obtained from the FEA simulations. On this platform, the dispersions were resolved when the cells have been seeded with a density near the electrode surface of above
300 cells $/ \mathrm{mm}^{2}$. The intensity of the dispersion increased as the single MCF10A cells aggregate over the 7 day assay (figure 5B and table 1). This increase coincided with the rise in the low frequency permittivity of the cell sample. Similar to the FEA simulation, as the cell cluster size increased, there was an increase in the low frequency permittivity and the dispersion intensity. There are statistically significant differences in the dielectric parameters between days 0 and 2 and for days 2 and 5 (Table 1). Notably, there is little difference between the dielectric parameters on days 5 and 7. A cause could be cell diffusion from the matrigel and adhesion onto the electrode surface, which would compromise electrode resolution; however, daily microscope inspection ensured the electrodes remained clear of cells. Instead, the lack of change in the dielectric spectra is most likely due to a slowdown in cell

Table 1 The dispersion intensity $\Delta \varepsilon$ and characteristic frequency $f_{c}$ from days 0-7 of the cell aggregation assay. The cells are seeded onto the platform on day 0 .

\begin{tabular}{|c|c|c|c|c|}
\hline & Day 0 & Day 2 & Day 5 & Day 7 \\
\hline $\begin{array}{c}\Delta \varepsilon \\
\left(10^{4}\right)\end{array}$ & $0.67 \pm 0.05$ & $1.21 \pm 0.08$ & $1.89 \pm 0.12$ & $1.99 \pm 0.07$ \\
\hline $\begin{array}{c}f_{c} \\
\left(10^{4}\right)\end{array}$ & $8.23 \pm 0.13$ & $3.98 \pm 0.06$ & $2.45 \pm 0.01$ & $2.31 \pm 0.03$ \\
\hline
\end{tabular}

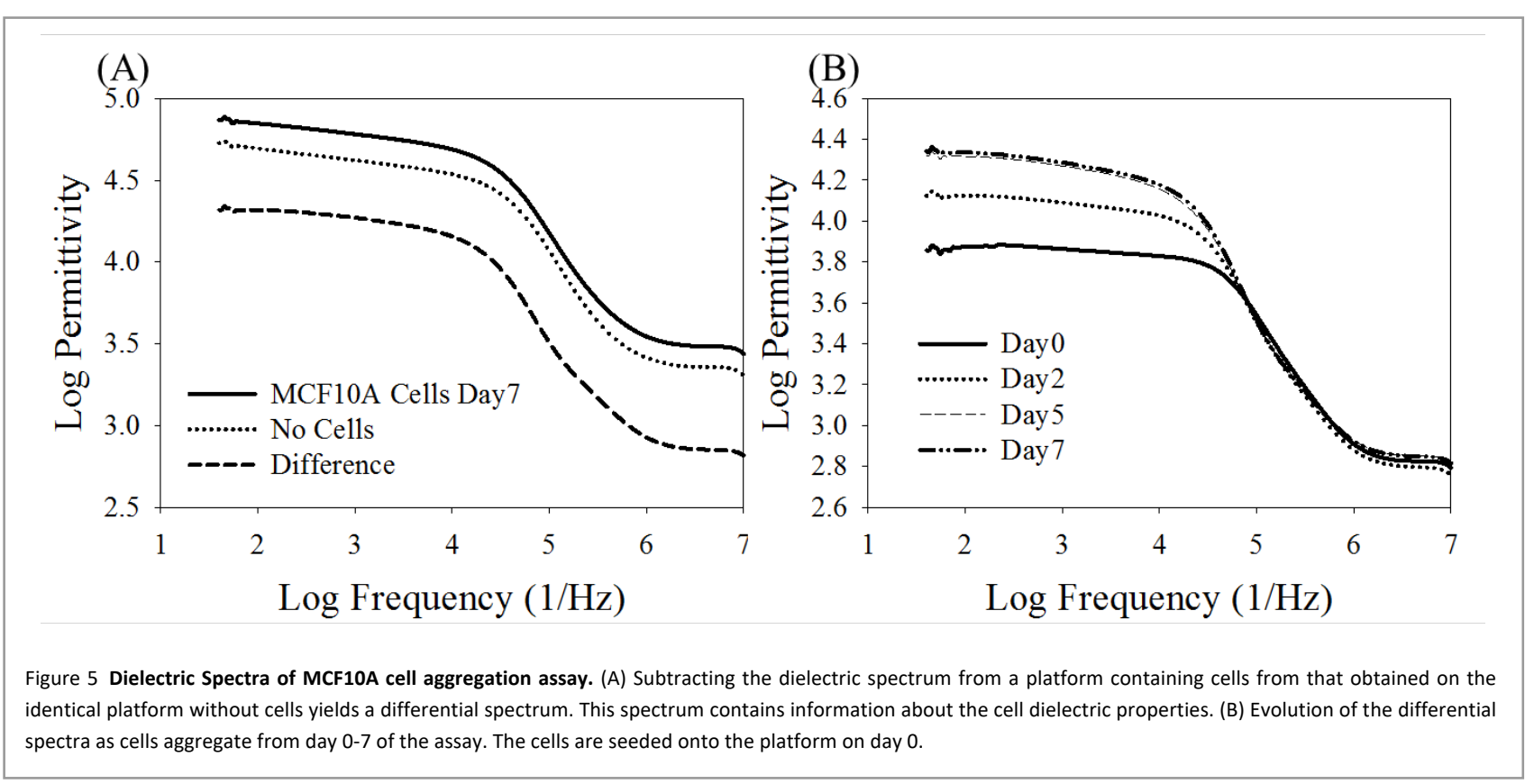


aggregate growth several days into the assay. Meanwhile, the dielectric spectra obtained from the control dish containing a cell-free matrigel layer remained relatively unchanged throughout the assay. This confirms that contribution from the cells dominate shifts in the dielectric spectra.

\section{Discussion}

By fitting the experimental dielectric spectra to the Cole-Cole equation, we demonstrated that the dispersion intensity $\Delta \varepsilon$ and the characteristic frequency $f_{c}$ are related to cell aggregate growth. These dielectric parameters are valuable to quantitatively monitor cell aggregation in a non-invasive manner. The results parallel those from a previous computer simulation study of cell aggregation, in which the low frequency permittivity increased while reducing the cell separation distance, until the cells were adhering ${ }^{19}$. This effect was due to the net contribution of induced dipoles from adjacent cells, increasing polarizability and hence permittivity ${ }^{19}$. Thus, this effect accounts for the increase in the dispersion intensity we observed during cell aggregate formation. In addition, the decrease in the characteristic frequency $f_{c}$ which occurred during cell aggregation (table 1 ) was also observed in the aforementioned study.

This type of platform has many applications for in vitro monitoring of cell aggregates and may be expanded to other morphologically similar tissues. For example, the aggregation of cells into artificial tissues, such as islets, could be monitored with dielectric spectroscopy to identify critical milestones, such as the formation of gap junctions between cells ${ }^{20}$. These gap junctions play a significant role in insulin secretion within normal islets, hence their expression in regenerated islets is an important marker of functionality expression ${ }^{21}$. Additionally, the platform could be used to understand the electrophysiological aspects of tumour formation. The dielectric response of tumour cells to drugs could be assessed on this type of platform, in particular due to tumour aggregate volume changes, allowing rapid and facile screening of large quantities of candidate drugs for cancer. The underlying cellular and biochemical effects of positively identified drugs can be further analysed with traditional genomic, transcription factor and protein profile analyses. Furthermore, impedance spectroscopy platforms are capable of distinguishing MCF10A and more malignant MCF-7, MDA-MB-231 and MDA-MB-435 cell lines ${ }^{22}$. Therefore, in addition to assessing tumour cell drug response, there is potential to establish the malignancy level of cells using dielectric spectroscopy.

In its current layout, this platform is simple to assemble once the microelectrodes have been fabricated. Moving further, this platform represents a step in the direction towards dielectric spectroscopy monitoring of 3-dimensional cell cultures in microfluidic systems. With a few minor alterations, the planar electrodes can be easily integrated into the layer-by-layer fabrication required for microfluidic platforms. These platforms enable precise delivery of stimulants and drugs to cells under study, with the ability to rapidly change concentrations. The continuous flow of microfluidic devices simulates in vivo blood flow, which is appropriate for long-term tissue cultures. In addition, microfabrication technologies allow better control of the microenvironment, including localization of tissues and the extracellular matrix. The geometry of these microenvironments can increase the volume fraction of the cells, increasing the cell contribution to the dielectric response. Furthermore, with microfluidic perfusion, effluent can be collected to measure secreted molecules of interest. Another advantage of planar electrodes is that the sample remains visible throughout the assay. Therefore, visual feedback regarding activity within the assay is provided, giving information about cell density, cell migration and tissue morphology. By using transparent indium tin oxide electrodes on thin substrates, immunofluorescence imaging can be performed on the same samples subject to dielectric spectroscopy. Immunofluorescence imaging gives information about cell differentiation progression, phenotype and associated morphology, yielding a multi-parametric analysis when combined with dielectric spectroscopy.

\section{Conclusions}

The presented platform used dielectric spectroscopy to monitor MCF10A cell aggregation for 7 days in a 3-dimensional extracellular matrix culture. Dielectric spectroscopy, which provides continuous and non-invasive measurements, is advantageous for multi-day cell aggregation assays. Although 3dimensional cell cultures simulate in vivo conditions, they present several challenges for dielectric spectroscopy monitoring, including lack of measurement sensitivity and signal penetration into the culture. This platform overcomes these challenges by adopting a geometry that increases the cell contribution to the dielectric response. As demonstrated, the formation of MCF10A aggregates impacted the dielectric spectra and allows for elucidation of the associated variation in cellular dielectric character. These variations were quantified using dielectric parameters from the Cole-Cole equation. Because cell aggregation is a major feature of in vitro models of tumour formation and organogenesis, this type of platform can provide many applications in drug discovery and regenerative medicine.

\section{Acknowledgements}

We gratefully thank the Natural Science and Engineering Research Council of Canada (NSERC) -Discovery and CREATE in Integrated Sensor Systems (ISS) and Continuous Flow Science (CFS), Canadian Institute of Health Research (CIHR), and the Fonds Québécois de la Recherche sur la Nature et les Technologies (FQRNT)-Team Grant for their financial support.

\section{Notes and references}


1. L. A. Kunz-Schughart, J. P. Freyer, F. Hofstaedter and R. Ebner, Journal of Biomolecular Screening, 2004, 9, 273285.

2. G. Benton, H. K. Kleinman, J. George and I. Arnaoutova, International Journal of Cancer, 2011, 128, 1751-1757.

3. O. W. Petersen, L. Rønnov-Jessen, A. R. Howlett and M. J. Bissell, Proceedings of the National Academy of Sciences of the United States of America, 1992, 89, 9064-9068.

4. F. Hirschhaeuser, H. Menne, C. Dittfeld, J. West, W. Mueller-Klieser and L. A. Kunz-Schughart, Journal of Biotechnology, 2010, 148, 3-15.

5. K. M. Yamada and E. Cukierman, Cell, 2007, 130, 601-610.

6. J. M. Kelm and M. Fussenegger, Trends in Biotechnology, 2004, 22, 195-202.

7. J. Hilderink, S. Spijker, F. Carlotti, L. Lange, M. Engelse, C. van Blitterswijk, E. de Koning, M. Karperien and A. van Apeldoorn, Journal of Cellular and Molecular Medicine, 2015, 19, 1836-1846.

8. D. Gallego-Perez, N. Higuita-Castro, R. K. Reen, M. PalacioOchoa, S. Sharma, L. J. Lee, J. J. Lannutti, D. J. Hansford and K. J. Gooch, Biomedical Microdevices, 2012, 14, 779-789.

9. A. B. Bernard, C.-C. Lin and K. S. Anseth, Tissue Engineering Part C: Methods, 2012, 18, 583-592.

10. T. Das, L. Meunier, L. Barbe, D. Provencher, O. Guenat, T. Gervais and A.-M. Mes-Masson, Biomicrofluidics, 2013, 7, 011805.

11. W. Wang, K. Itaka, S. Ohba, N. Nishiyama, U.-i. Chung, Y. Yamasaki and K. Kataoka, Biomaterials, 2009, 30, 27052715.

12. F. Pampaloni, E. G. Reynaud and E. H. K. Stelzer, Nat Rev Mol Cell Biol, 2007, 8, 839-845.

13. F. A. Alexander, D. T. Price and S. Bhansali, IEEE Reviews in Biomedical Engineering, 2013, 6, 63-76.

14. K. M. Imbalzano, I. Tatarkova, A. N. Imbalzano and J. A. Nickerson, Cancer Cell International, 2009, 9, 7-7.

15. J. Debnath, S. K. Muthuswamy and J. S. Brugge, Methods, 2003, 30, 256-268.

16. K. Asami, Journal of Non-Crystalline Solids, 2002, 305, 268277.

17. P. Van Gerwen, W. Laureyn, W. Laureys, G. Huyberechts, M. Op De Beeck, K. Baert, J. Suls, W. Sansen, P. Jacobs, L. Hermans and R. Mertens, Sensors and Actuators B: Chemical, 1998, 49, 73-80.

18. K. Asami, Progress in Polymer Science, 2002, 27, 16171659.

19. A. Ron, N. Fishelson, N. Croitoriu, D. Benayahu and Y. Shacham-Diamand, Biophysical Chemistry, 2009, 140, 3950.

20. J. Daoud, K. Heileman, S. Shapka, L. Rosenberg and M. Tabrizian, Analyst, 2015, 140, 6295-6305.

21. N. L. Farnsworth and R. K. P. Benninger, FEBS letters, 2014, 588, 1278-1287.

22. A. Han, L. Yang and A. B. Frazier, Clinical Cancer Research, $2007,13,139-143$ 